The manuscript is a preprint and has been submitted for publication in PNAS. Please note that, the manuscript has not undergone peer review. Subsequent versions of this manuscript may have slightly different content. If accepted, the final version of this manuscript will be available via the 'Peer-reviewed Publication DOI' link on the right-hand side of this webpage. Please feel free to contact any of the authors. We welcome feedback! 


\title{
Variations in Earth's 1D viscosity structure in different tectonic regimes
}

\author{
Anthony Osei Tutu ${ }^{1}$ and Christopher Harig ${ }^{1}$ \\ ${ }^{1}$ Department of Geosciences, University of Arizona, Tucson, USA \\ This manuscript was compiled on January 12, 2022
}

\begin{abstract}
Earth's long-wavelength geoid provides insights into the thermal, structural, and compositional evolution of the mantle. Historically, most estimates of mantle viscosity using the long-wavelength geoid have considered radial variations with depth in a symmetric Earth. Global estimates of this kind suggest an increase in viscosity from the upper mantle to lower mantle of roughly $2-3$ orders of magnitude. Using a spatio-spectral localization technique with the geoid, here we estimate a series of locally constrained viscosity-depth profiles covering two unique regions, the Pacific and Atlantic hemispheres, which show distinct rheological properties. The Pacific region exhibits the conventional Earth's 1D rheology with a factor of roughly 80-100 increase in viscosity occurring at transition zone depths $(400-700 \mathrm{~km})$. The Atlantic region in contrast does not show significant viscosity jumps with depth, and instead has a near uniform viscosity in the top $1000 \mathrm{~km}$. The inferred viscosity variations between our two regions could be due to the prevalence of presentday subduction in the Pacific and the infrequence of slabs in the Atlantic, combined with a possible hydrated transition zone and midmantle of the Atlantic region by ancient subduction during recent supercontinent cycles. Rigid slab material within the top $800 \mathrm{~km}$, with about $90 \%$ Majoritic garnet in the form of subducted oceanic crust, coupled with unique regional mantle structures, may be generating a strong transition zone viscosity interface for the Pacific region. These effective lateral variations in mantle viscosity could play a role in the observed deformation differences between the Pacific and Atlantic hemispheres.
\end{abstract}

Mantle viscosity | spatiospectral localization | geoid | subduction

$\mathbf{T}$ he viscosity of Earth's mantle is fundamental to the operation of convection and plate tectonics, and as a result, it has been extensively studied over the past several decades. Many studies have used the long wavelength $(l=$ $2-3)$ geoid and mantle flow calculations to explore the radial viscosity and density structures of the mantle (1-6). Hager and Richards et al.(7) showed that about $90 \%$ of the observed longwavelength geoid signal can be explained with a model based on flow driven by seismically derived mantle density. The geoid together with other geophysical processes (post-glacier rebound (8), dynamic uplift (9), plate motions (10), etc.) have been used to constrain both the relative and absolute viscosities of the mantle.

Most inferences of Earth's long-wavelength mantle viscosity structure rely on a spherically symmetric representation of viscosity [radial variation only] (11). This assumption permits a regional constrained viscosity-depth profile to be extended and applied over the entire globe. For example, authors have solved for the depth-dependent viscosity structure based on a regional waxing and waning of ice sheets in the past 20,000 years (12). Such regionally constrained viscosity profiles may at best be representative of the local viscosity-depth variations beneath the glaciated area and immediate surroundings (13), and perhaps not applicable to other areas of the globe.

Here we use a new method to develop the new large-scale regional estimates of the mantle's long-wavelength radial viscosity structure using Earth's static geoid. These estimates illustrate how strong regional mantle heterogeneities (or lack thereof) influence the regional radial viscosity structure. We employ a spatio-spectral localization technique (Slepian basis functions - see Materials and Methods) to study any potential differences that may exist between global and regionally constrained radial viscosity structures. We use a Bayesian inversion approach to solve for local mantle viscosity profiles in two unique regions of the present-day mantle. The first region covers the circum-Pacific, encompassing most of the present-day active subduction systems in and around the $\mathrm{Pa}$ cific plate (Fig.1a ). The second region covers an area with predominately less active or recently active subduction zones centered in the Atlantic Ocean.

The regional viscosity inversion is used to highlight the importance of local mantle heterogeneities, such as subduction, slabs and other regional geodynamic processes, to mantle radial viscosity characteristics. Large-scale mantle flow studies generally invoke subducted slab structure and rheology to explain lateral viscosity variations $(14,15)$. There is no established relation on the plausible influence of slabs rheology to the radial mantle viscosity structure. Slabs seen in seismic tomography models occupy a low volume of the overall mantle. Rigid slab remnants are mainly concentrated in the upper mantle and the uppermost lower mantle where they make up a relatively larger volume (16-18). The complexity of slabs geometry with the different styles and stages of subduction (17), concentrated in specific regions and depths of the mantle

\section{Significance Statement}

The surface and internal structures of Earth move on a time scale of tens to hundreds of million years. The slow motion of continents as shown by satellite observations is dependent on the viscosity of Earth's interior. We use mathematical methods and computer simulations to study viscosity/strength as a function of depth in two regions: the Pacific and Atlantic hemispheres. Our calculations show that the Pacific region of Earth's interior is relatively stronger than the Atlantic region. We interpret these differences as the results of the spatial distribution of subduction, where oceanic lithosphere is recycled into mantle.

A.O.T. and C.H. designed research, performed research, and wrote the paper.

The authors declare no conflict of interest.

${ }^{2}$ To whom correspondence should be addressed. E-mail: oseitutuarizona.edu.
23 

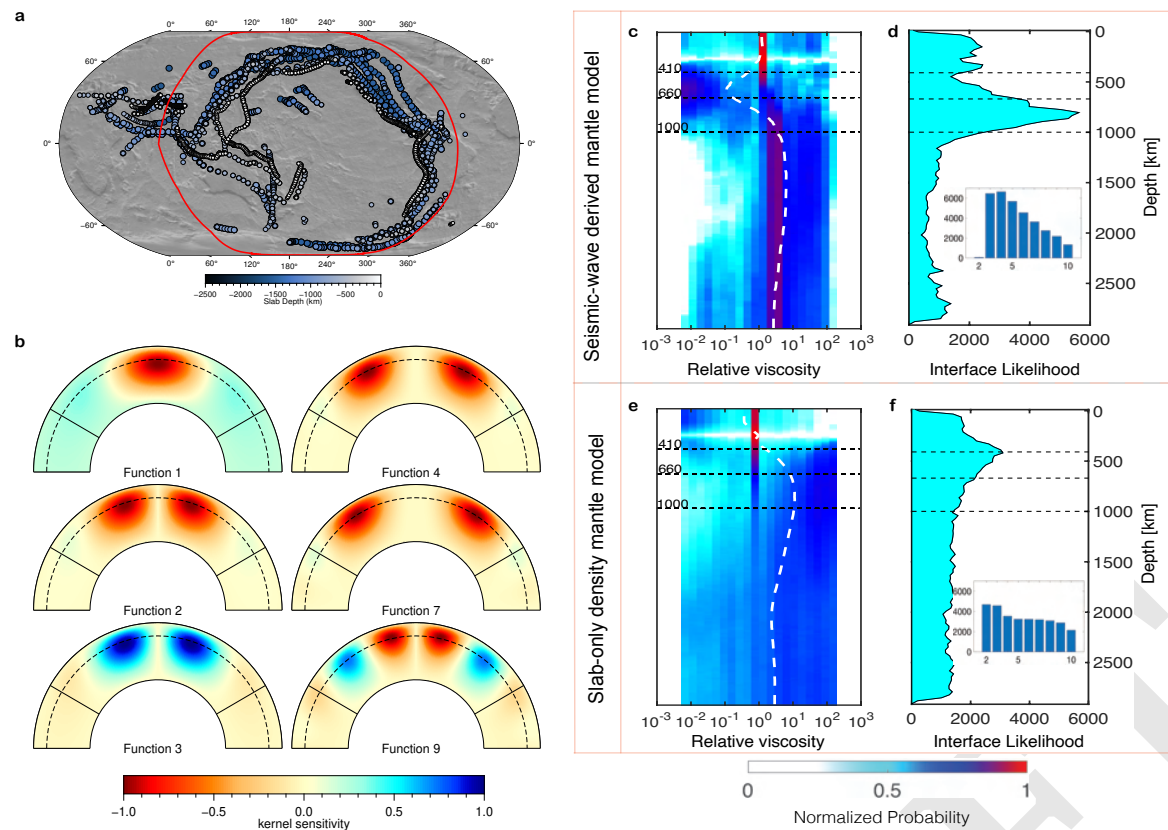

Fig. 1. a) An outline (red line) of our Pacific region for the local constrain layered mantle viscosity inversions showing locations and depths of present-day slabs distribution (22) in the mantle. b) Local sensitivity dynamic geoid kernels with an iso-viscous mantle. Shown is a cross-section along $0^{\circ}$ and $180^{\circ}$ in the northern hemisphere from the surface to the core mantle boundary. The kernels have azimuthal dependence and as such will have different manifestations at different azimuths. The kernels are localized to a $50^{\circ}$ spherical cap, denoted by black lines connecting the surface to the core mantle boundary and the dash lines show the $670 \mathrm{~km}$ depth. The bandwidth of the basis is $l=9$. Functions are ranked by concentration within the region, and shown are functions 1, 2, 3, 4, 7, and 9. Here the kernels are normalized by their maximum absolute value. The kernels can be localized in both regular and irregular (red outline) spherical caps. c) Layered mantle viscosity solutions from global large-scale mantle flow for spherical harmonics degrees $l=2$ to 3 using a seismically-derived mantle model (19) (c-d) with constant scaling and plate reconstruction slab-only mantle model (21) (e-f). Panels c and e show 2D histograms of the posterior probability distributions of viscosity with depths expressed as normalized probability and the white dash lines giving the mean relative viscosity profiles. Panels $\mathbf{d}$ and $\mathbf{f}$ show resulting mantle-viscosity interfaces distribution with the corresponding inset histograms giving the number of layers for each solution.
(Fig.1a and Supplementary Information fig.S1b), may suggest local radial viscosity profiles that are unique to regions of the mantle. Mantle viscosity is known to be dependent on both chemical (e.g., major mineral assemblage such as Ferropericlase and Bridgmanite) and physical (e.g., temperature, pressure, deformation mechanism, strain rate, grain size) properties. We consider two different scenarios of the mantle structure. We use mantle density models based on seismic tomography [SEMUCB-WM1 (19) and S362ANI+M (20)] referred to herein as Seismic-wave derived mantle models. Our second mantle scenario, the Slab-only density mantle model, is based on a plate/slab reconstruction model [STB00 (21)].

Global constrained radial viscosity solution. To better quantify the significance of regional mantle heterogeneities to radial viscosity, we first infer a series of global constrained viscosity profiles and verify our solutions with recent published studies (23). In each case we use a probabilistic sampling solution method (see Materials and Methods) to synthesize the global geoid fields and compare with the respective observed timeinvariant geoid signal from GRACE (24) satellite data to infer the global viscosity structure. We focus on long ( $l=2$ to 3 ) and intermediate $(l=4$ to 9 ) spherical harmonic wavelengths of the geoid. The posterior distribution of our $l=2$ to 3 globally constrained relative viscosity solution (Fig. 1c) based on seismically derived mantle structure predicts a low-viscosity transition zone with strong upper mantle (i.e. above $410 \mathrm{~km}$ ) and lower-mantle viscosities. There is roughly a one order of magnitude viscosity increase between the transition zone and the lower mantle. The viscosity increase between 670 $\mathrm{km}$ and the lower mantle is supported by a high probability mantle interface (Fig. 1d). Our globally constrained longwavelength ( $l=2$ to 3 ) viscosity structures, using seismically derived density models, are consistent with past large-scale mantle flow studies $(3,5,23)$. The $l=2-3$ viscosity inversion experiments with other seismic tomography models using either single parameter (Supplementary Information fig. S5a-b) or depth-dependent (Supplementary Information fig. S5e-f) seismic velocity-to-density scaling show similar mantle viscosity-depth characteristics.

For our slab-only mantle density model (21), the global $l=2-3$ viscosity solution, shows a relatively strong transition zone (Fig. 1e) compared to the prediction using the seismicderived mantle model (e.g., Fig. 1c). Note that for the slab-only mantle, we are assuming a mantle convection style which depends on only subduction and slab material. Hence, our prediction of a strong transition zone (Fig. 1e-f) is not surprising in the absence of hot buoyant mantle material. The large accumulation of rigid slab material within the transition zone and above $1000 \mathrm{~km}$ depth (17) maybe a contributing factor generating a stiff viscosity interface. This may also suggest a non-negligible long wavelength component of slabs' influence on viscosity-depth variations.

The set of intermediate wavelengths ( $l=4$ to 9$)$ globally constrained viscosity profiles, shows predominately the sensitivity of geoid data to slab remnants (1). Both the seismicwave derived model and the slab-only mantle density models (Supplementary Information fig. S4a-b and S4c-d) predict a weak asthenosphere channel, followed by a stiff transition zone. Panasyuk and Hager et al.(4) have suggested a similar layered mantle viscosity structure showing a strong transition zone, using a combination of slab densities in the upper mantle and seismic-based densities for the lower mantle. Our results show a high probability viscosity-and-mantle interface around the $410-\mathrm{km}$ depth with a viscosity jump of more than 2 orders of magnitude between the asthenosphere (upper mantle) and 

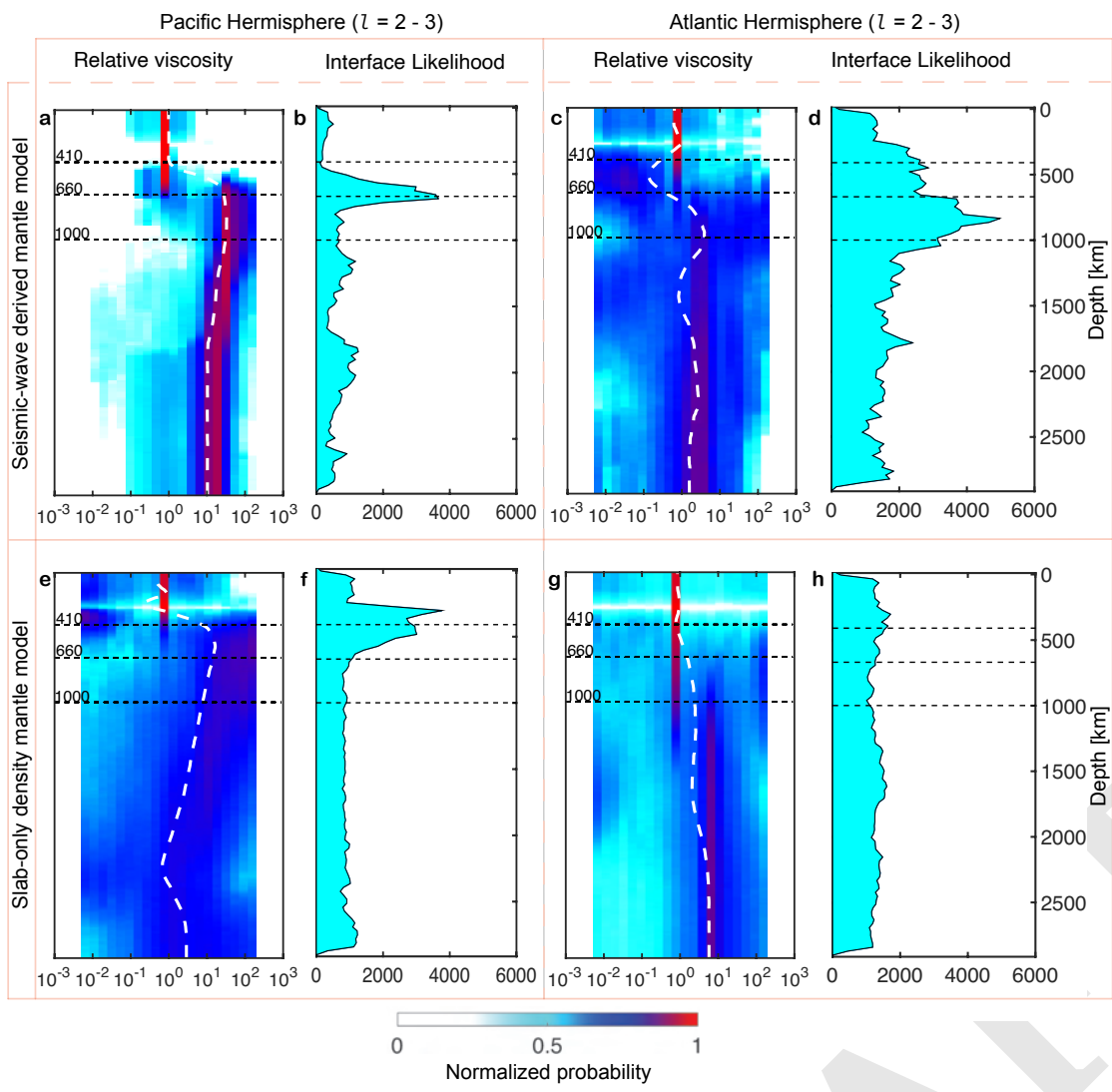

the mid-mantle. Such values of relative viscosity $(c a .300)(7)$ between the asthenosphere and lower mantle is required to fit the observed slab geoid.

\section{Local constrained radial viscosity solu-} tion. Using a Slepian localization technique (Fig.1b, see Materials and Methods), we derive local geoid signals (i.e. Pacific and Atlantic hemispheres) and infer a viscosity solution for each region. In each case, we consider the same mantle density models and geoid spectrums (i.e. $l=2$ to 3 and $l=4$ to 9 ) used in the global solutions above. The resulting regional viscosity structures show distinct differences in the top $800 \mathrm{~km}$ of the mantle, particularly across the mantle transition zone. By comparing the $l=2-3$ inferred viscosity structures for the Pacific (Fig.2a-b and 2e-f) to the Atlantic (Fig.2c-d and $2 \mathrm{~g}-\mathrm{h}$ ) regions, we see the unique influence of the respective local mantle structures.

In the Pacific domain, we find some degree of stiffness in the vicinity of the transition zone (Fig. $2 a-b$ and $2 e-f$ ). Conversely the Atlantic regional solutions, which have little/noslab heterogeneities within the top $800 \mathrm{~km}$ of mantle, show no such stiff viscosity interface. Rather we infer a relatively lowviscosity transition zone (Fig. $2 c-d$ and $2 g-h$ ). A similar phenomenon is also observed for the $l=4-9$ regional viscosity inversions shown in Fig. 3b for the Pacific (blue lines) and Atlantic (red lines) hemispheres. Maps showing the respective local geoid anomalies of the Pacific and Atlantic regions for $l=2-3$ and $l=4-9$ are provided in the supplementary information (fig. S9). We employed a second seismic model S362ANI+M (20) and repeat our regional calculations (Fig.3, solid lines), which show similar results for the Pacific and Atlantic local inversions (Supplementary Information fig. S8).

Localizing around and away from the subduction systems (e.g., Red outline Fig.1a) shows the apparent effect of the local mantle structures. The presences of slab heterogeneities within the Pacific local mantle may be the controlling factor giving rise to the stiff transition zone at long (Fig.3a, green region) and intermediate wavelengths local viscosity solutions (Fig. $3 \mathrm{~b}$, green region). While phase changes and mantle composition predominantly have been proposed to dictate the characteristics of the transition zone viscosity (25), our results suggest additional crucial contributions from the local thermal/density structures.

Our understanding and interpretations of the mantle radial viscosity structure are mostly centered on the rheological properties of the global ambient mantle. The new approach used here allows us to explore the potential influence of regional mantle densities/temperatures to viscosity-depth variations, which may be a challenge in large-scale mantle flow studies. The prediction of stiff (Pacific, Fig. 3a-b blue lines) and weak (Atlantic, Fig. 3a-b red lines) transition zone viscosities, are at first-order due to the presence and absence of slab remnants within each local mantle. This finding illuminate past conclusions (e.g., $(3,5,27,28))$ on mantle transition zone viscosity profiles, which relied on the mantle hot anomalies. The coupled hot mantle and cold slabs with phase transitions may be playing an equal role on the exact amplitude of the transition zone rheology. We would expect to predict similar viscosity 

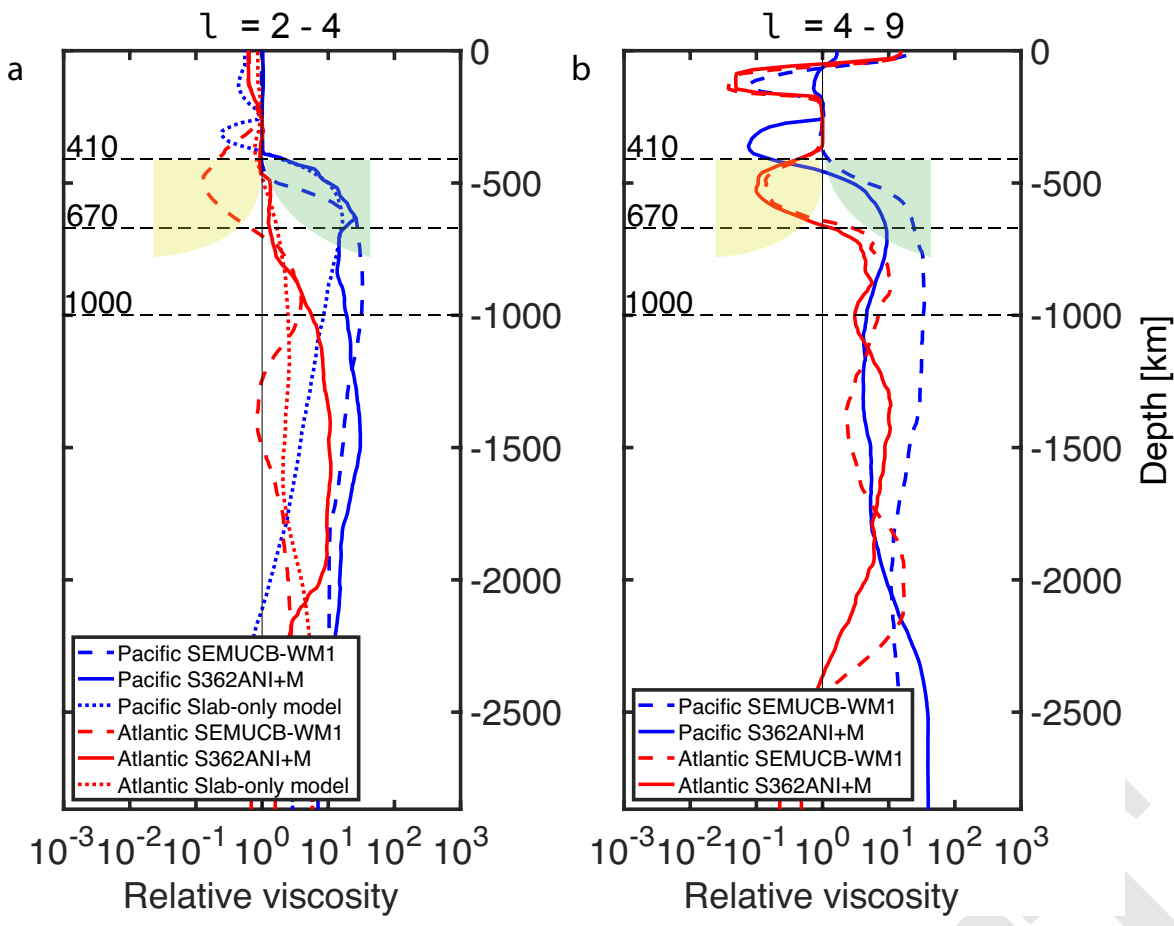

Fig. 3. Plots showing a) the averages of longwavelength ( $l=2-3)$ local viscosity solutions based on seismically-derived mantle model SECUMB-WM1(19) (dashed), S362ANI + M (20) (solid) and slab-only mantle model (21) (dotted) for the Pacific (blue) and Atlantic (red). b) Averages of intermediate-wavelength $(l=4-9)$ local viscosity solutions based on seismically-derived mantle model SECUMB-WM1(19) (dashed), S362ANI + $M$ (20) (solid) for the Pacific (blue) and Atlantic (red). The yellow and green shaded regions show the respective Atlantic and Pacific viscosity solutions interface preference in the top mantle.

profiles for the two regions per our assumption of spherical symmetry of global constrained viscosity profiles. Our inferred viscosity-depth differences suggest that slab rheology may be as important to the layered mantle viscosity as it is to lateral viscosity variations, especially in the top $800-\mathrm{km}$ of the mantle.

Subducted oceanic crust in the mantle transition zone contains garnet-rich layers (Majorite). These layers have been suggested (29) as a major contributing factor for the strong transition zone viscosity. The prediction of low-viscosity interfaces with our less/no slabs region (Fig. 3 red profiles) versus the stiffness obtained with the slabs dominated Pacific local mantle (Fig. 3 blue profiles) tends to support this observation. The presence of other garnet-rich composition within the mantle transition zone in the form of either pyrolite or piclogite (i.e. peridotite and eclogite) will also influence the Pacific and Atlantic local viscosity profiles. But the high volumetric ratio (about 90\% (29)) of garnet constituents in subducted oceanic crust and cold slabs structures within our Pacific region of the mantle will likely account for most of the extra hardness within the transition zone. The debate surrounding stiff $(27,30)$ or weak transition zone (6) dates back several decades among large-scale mantle flow studies. This discrepancy may be due to the intrinsic deficiencies among the global seismic models used for those studies, since slabs are resolved differently in various seismic models. Our viscosity localization experiments may shed light on the debate of the origins of hard and soft transition zone viscosity.

Our inference of Atlantic region low viscosity interface may have additional influence of a wet transition zone and the top of the lower mantle by slabs dehydration (31) from the Pangea subduction system. The presence of water in the upper mantle has been shown to affect viscosity and as a source of melting generation (29). Ohtani etal., (31) recently showed as slabs descends into the mantle they hydrate the mantle layers above (Fig. 4). Their experiment suggest that dense hydrous magma may form at the base of the upper mantle and move upward as slabs dehydrate. As cold hydrated slabs pass the transition zone into the lower mantle either by mantle suction or gravitational collapse fluids/volatile-rich magmas may generate due to the wide variation in water content between mineral composition of the mantle transition zone and the lower mantle. Though this phenomenon is mostly likely to be observed in the Pacific region with the present-day subduction. Paleo-subduction studies (e.g.,(26)) constraining longitudinal positions of past oceanic subduction zones showed the Atlantic mantle has experienced a period of active subduction comparable to the present-day Pacific subduction systems. van der Meer etal., (26) mapped out the current locations of slab remnants in the mid and lower mantle using plate reconstruction and seismic model (Supplementary Information fig. S11). Their analysis showed that most lower mantle slabs materials are concentrated in the Atlantic region, for example the Atlantis, Georgia Island, Algeria, Farallon plates, etc (Fig. 4a). It's possible such volatile-rich mantle depths induce by past Pangea subduction may persist over $100-200$ Myr (Fig. 4c), which will affect our Atlantic viscosity inference.

A number of authors have suggested the presence/remnants of distinct heating (or temperatures) within the respective local mantles (32-34) considered in our current study. According to Le Pichon et al.(33), the assemblage and stationarity of the supercontinent Pangea with peripheral subduction systems led to a thermally insulated mantle. A recent study by Karlsen etal., (34) of the two hemispheres (Pacific and Atlantic), has suggested a temperature deficit of about $50 \mathrm{~K}$ with the Pacific region been colder. We explore this by localizing in central Pacific excluding all slab to infer viscosity and compared with inversion focusing on western Pacific (see Supplementary Information Fig. S10). The central Pacific mantle gave a less stiff upper mantle compare to the western Pacific region with old slabs suggestion this temperature deficit may have less 
a

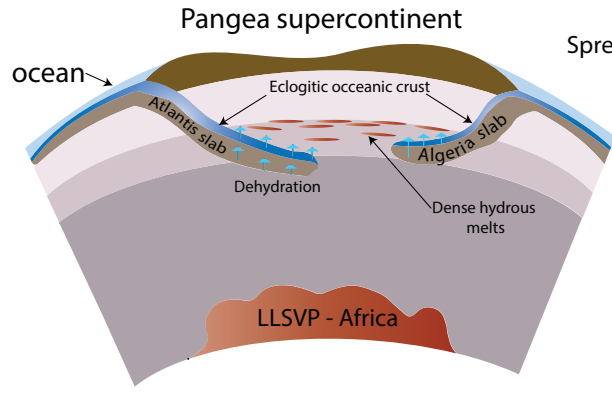

C

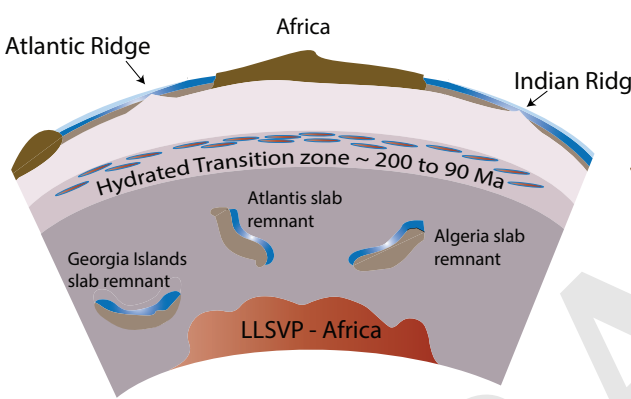

Present day Atlantic Hemisphere b

Pacific Region 300 to $150 \mathrm{Ma}$
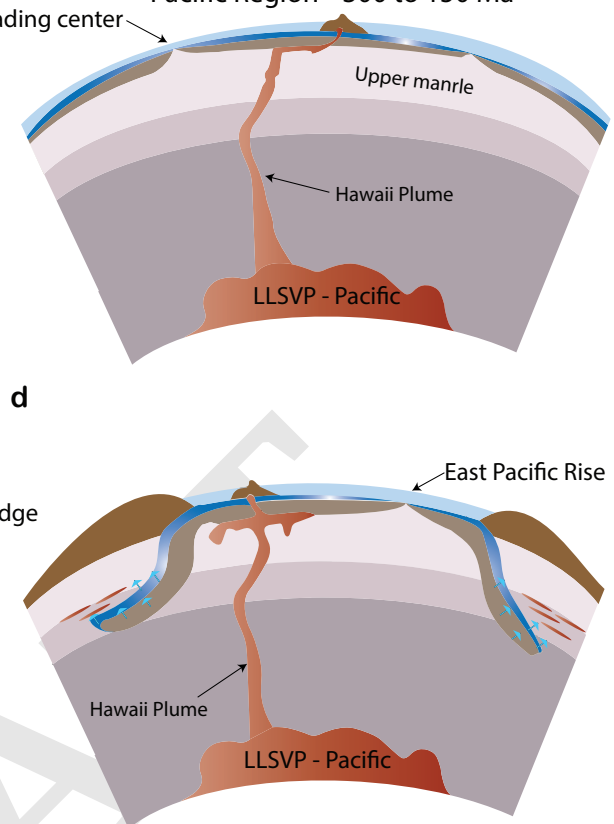

Present day Pacific Hemisphere

e

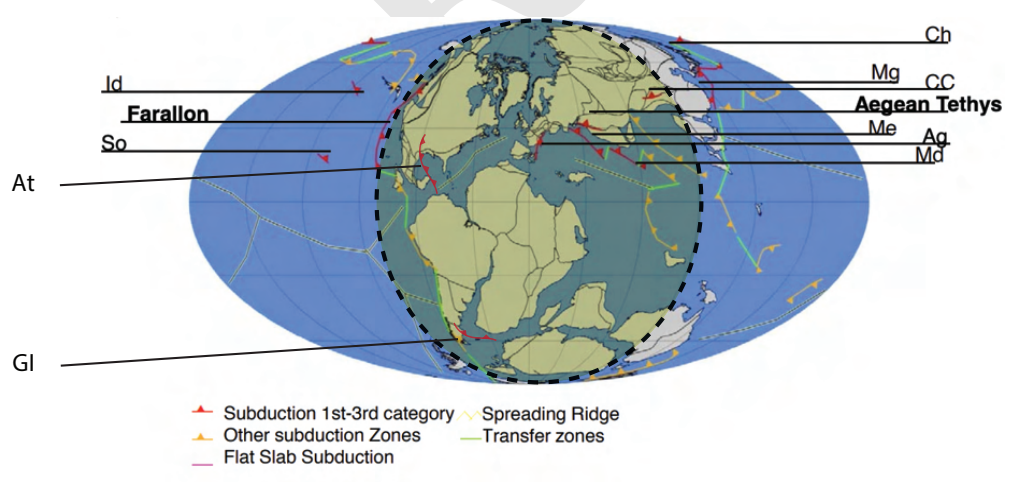

Fig. 4. Schematic illustration of a possible hemispheric difference between (a) Atlantic and (b) Pacific regions during the Jurassic and Early Cretaceous eras showing the peripheral subduction of the Pangea supercontinent and spreading centers respectively. (c) Present-day Atlantic hemisphere showing a possible hydrated transition zone and/or top of the lower mantle from past subduction with remnants of the Atlantis, Algeria and Georgia Island slabs in the deep mantle. (d) Pacific region showing present-day subduction systems and the Hawaii plume. c) A Paleo-Geographic map with the longitudinal position of past oceanic subduction zones modifies after van der Meer et al., (26) depicting the likely position of the Ag - Algeria, CC - Central China, Ch - Chukchi, Id - Idaho, Me - Mesopotamia, At - Atlantis, Mg - Mongolia, Gl Georgia Islands, So Socorro, Md - Maldives slabs. The overlying yellow shade with dash black outline shows the approximate Atlantic region for the local viscosity inversion with our spatiospectral localization technique. 
influence on our results compared to subducted oceanic plate.

In summary, we suggest that regional mantle structures have a unique control on the local viscosity inference and likely the global viscosity profile. Especially within the top $800 \mathrm{~km}$ of the mantle, slabs heterogeneities show non-negligible influence on the viscosity-depths variations in the mantle transition zone. There may be additional contribution from a difference in the regional mantle hydrations. Our findings put a first-order constraint on the long-wavelength lateral viscosity variations within the top half of the mantle. This is characterized by the presence of a strong transition zone in and around predominantly slabs and subducting regions, combined with a comparatively low-viscosity transition zone. The inferred significance of slab rheology to the depth-dependent viscosity structure suggests global profiles created with the assumption of spherically symmetric mantle flow driven only by ambient density should be interpreted cautiously in regional settings, even at large scales.

\section{Materials and Methods}

We focus on regional constraints of Earth's 1D viscosity structure in two tectonic regimes, using a convective geoid model based on seismic and slab density models. We analyzed the geoid data in the spectral ranges $l=2$ to 3 (long-wavelengths) and $l=4$ to 9 (intermediate wavelengths). The intermediate range (i.e. $l=4-9$ ) of the geoid has been shown to be more sensitive to density variations due to subducted slabs (1), whereas the long wavelength geoid $(l=$ $2-3)$ is sensitivity to lower mantle density structure.

We use local geoid kernels based on Slepian basis functions (35) for the regional viscosity inversions. In most regional geophysical data analysis based on global data, one of the important issues that often needs further consideration is spectral leakage and/or contamination of the data signal in the region of interest (35). In our case, it is very important to understand the extent of depth contributions from the local mantle heterogeneities, and explicitly seek to minimize any leakages with respect to depth and lateral influences. For example, considering an iso-viscous mantle, we can test the local sensitivity kernels $(\mathrm{L}=1-30$ in FigS. 1 e and $\mathrm{L}=1-9$ in Fig.1b main text) for sensitivity to a sub-surface anomaly in a location of the mantle to show the robustness of our method at depth and lateral extent for different bandwidths.

Local and global geoid kernels: Forward modeling. We constrain local mantle viscosity and density structure for two unique regions (i.e., Pacific and Atlantic hemispheres) using a Bayesian probabilistic inversion with local non-hydrostatic geoid data. We analyze the geoid data in the spectral ranges $l=2$ to 3 and $l=4$ to 9 .

The spectral synthesis of regional geophysical signals from global spherical harmonics coefficients over a local region is often done using a localization technique such as radial basis functions, wavelets (36), or point masses (37). Here we use Slepian basis functions (35) to examine the local geoid in our regions. A number of previous studies have employed Slepian localization analysis, for example, to map Greenland Ice mass balance (e.g., $(38,39))$ or to study earthquake gravitational changes from the GRACE gravity data(e.g.,(40)) Each Slepian basis function constitutes a linear combination of the spherical harmonics on a sphere, with the specific combination determined by an optimization over the local region of interest. A detailed formulation can be found in Wieczorek and Simons et al., (35) and Simons et al., (41) with a practical treatment presented in Simons (42).

Our localization procedure combines Slepian basis functions with the non-linear Green's response functions (known as geoid kernels) $\mathcal{G}^{l}(r, \eta(r))$ representing the dynamic contribution of Earth's mantle to the anomalous geoid at the surface. The global dynamic geoid anomaly is calculated as

$$
\delta V_{l m}(\mathbf{S})=\frac{4 \pi G S}{2 l+1} \int_{c}^{S} \mathcal{G}^{l}(r, \eta(r)) \delta \rho_{l m}(r) d r
$$

where $\mathrm{G}$ is the gravitational constant, and $l$ and $m$ are the spherical harmonic degree and order respectively. $r$ denotes the mantle radius between the surface $(S)$ and the core mantle boundary $(c)$. We perform a Bayesian inversion during which each Markov-chain Monte Carlo (MCMC) step, the proposed relative viscosity structure $\eta$ is used to derive the geoid response function, which is convolved with the mantle lateral density heterogeneities $\delta \rho_{l m}(r)$ in spherical harmonics to synthesize the global geoid anomaly signal in spectral domain $\left(\delta V_{l m}(\mathbf{R})\right)$.

To build our Slepian basis (and examine the local geoid signal) we use the outline of the local region of interest $R$, for example the red outlines in fig. 1a of the main text for our Pacific region (see Supplementary Information fig. S2 and fig. S3 for Pacific and Atlantic regions) to integrate the products of the spherical harmonics $Y_{l m}(r)$ as

$$
\int_{R} Y_{l m} Y_{l^{\prime} m^{\prime}} d \Omega=D_{l m, l^{\prime} m^{\prime}} .
$$

The 'localization kernel' $\mathbf{D}$ is then decomposed in a matrix eigenvalue equation,

$$
\sum_{l^{\prime}=0}^{L} \sum_{m^{\prime}=-l^{\prime}}^{l^{\prime}} D_{l m, l^{\prime} m^{\prime}} g_{l^{\prime} m^{\prime}}=\lambda g_{l m},
$$

where the Slepian basis functions $g_{l m}$ are the eigenfunctions, and the eigenvalues $0 \leq \lambda \leq 1$ represent the degree of concentration of each function within the region (41). We show sets of sensitivity maps of the Slepian basis functions of well-concentrated functions for the Pacific (Fig. S2) and Atlantic (Fig. S2) hemispheres with $\lambda>0.5$. We have applied our Slepian localization technique in a joint inversion analysis of postglacial rebound and convection data to study the western shallow and eastern cratonic upper mantle viscosity structures of North America continental area (43).

We use the PREM (44) model as our depth-dependent reference density of the mantle with the geoid kernel estimation and neglect mantle compositional variations so not to interfere with any distinct regional viscosity difference we may infer. We derive the mantle density structures from two seismic tomography models [SEMUCBWM1 (19) and S362ANI+M (20)] following the relation $\delta \rho=\frac{\partial \ln \rho}{\partial \ln V_{s}}$. We test both single parameter (0.35) and depth-dependent seismic velocity-density scalings (45). We remove density heterogeneities in the top $300 \mathrm{~km}$ in oceans and continents due to the complex and compositional origin of continental roots. In addition we employ the geodynamically derived slab density model STB00 (21), which is based on a tectonic plate reconstruction. Employing a wide range of mantle density models will ensure that our resulting local and global viscosity-depth characteristics are not data dependent or artificial. Forte and Peltier (46) showed the implications on the choice of mantle density structure for large-scale mantle flow viscosity inferences. They concluded that the choice of mantle internal density structure used to infer the radial mantle viscosity structure plays a major role in the resulting viscosity structure due to the sensitive nature of the viscosity profile to the mantle density model. This makes it appropriate to test different density models and also to take advantage of the most recent seismic tomography with improved detail and resolution.

Transdimentional Bayesian Inversion. Our Bayesian inversion approach is a transdimentional, hierarchical, Markov-chain Monte Carlo (MCMC) inversion similar to the method of Rudolph et al., (23), used to infer global depth-dependent mantle viscosity structure. This procedure allows for the simultaneous inversion of the model data uncertainties $(47,48)$, making it suitable for nonlinear geophysical structures $(47,49)$, specifically in the case of non-unique solutions of mantle viscosity. At each step of the Markov Chain Monte-Carlo iterations a relative viscosity structure is defined by proposing a candidate viscosity value and/or depth interface (Birth, Death, Move and Value change). A fixed viscosity layer interface is set at the base of the lithosphere chosen at $250 \mathrm{~km}$.

The fifth step for the MCMC, which constitutes the hierarchical method with equal probability as the other steps, is a Noise step which accounts for possible data uncertainties. Each MCMC step is 
randomly selected with equal probability and a step-wise increase in mantle layers. Our probability solution relies on the Metropolis Hastening algorithm to decide at each step, whether to accept or reject the proposed solution based on a minimization given as

$$
\min \left[1, \frac{L\left(D \mid G^{\prime}\right)}{L(D \mid G)} \frac{n+1}{n^{\prime}+1}\right] .
$$

The likelihood probability function is defined as

$$
L(D \mid G)=\frac{1}{\sqrt{(2 \pi)^{n_{l m}}\left|M_{D}\right|}} \exp \left[-\frac{\Phi(G)}{2}\right] .
$$

Here, the Mahalanobis distance misfit function $M_{D}$ measures the fitness of both the amplitudes and pattern between the observed geoid and the synthetic geoid at each iteration step, which is given as $\Phi(G)=R^{t} M_{D}^{-1} R$ and the residual as $R=d-G(m)$ respectively. The $M_{D}$ is the covariance matrix and in our case we consider only a diagonal matrix to invert for model uncertainties employing a Gaussian noise distributions prior. At each step of the inversions, a new geoid response function is derived based on the perturbed viscosity and depth sampled from a prior distribution for each of the MCMC steps.

ACKNOWLEDGMENTS. We thank the University of Arizona's High Performance Computing center for granting us the free computational resources used for this study. We thank Bernhard Steinberger, Vedran Lekić and Raj Moulik for helpful discussions, and Shijie Zhong who read an early version of this manuscript. Figures were created with GMT and Matlab, and the accompanying numerical code used for our calculations with be made available on the following Github page (https://github.com/oseitutu) and (https://github.com/harig00). Funding: This work was made possible by NASA grant NNX17AE18G to $\mathrm{CH}$.

1. Hager BH (1984) Subducted slabs and the geoid: Constraints on mantle rheology and flow. Journal of Geophysical Research: Solid Earth 89(B7):6003-6015.

2. Richards MA, Hager BH (1984) Geoid anomalies in a dynamic earth. Journal of Geophysical Research: Solid Earth 89(B7):5987-6002.

3. Forte AM, Woodward RL, Dziewonski AM (1994) Joint inversions of seismic and geodynamic data for models of three-dimensional mantle heterogeneity. Journal of Geophysical Research: Solid Earth 99(B11):21857-21877.

4. Panasyuk SV, Hager BH (2000) Inversion for mantle viscosity profiles constrained by dynamic topography and the geoid, and their estimated errors. Geophysical Journal International 143(3):821-836

5. Steinberger B, Calderwood AR (2006) Models of large-scale viscous flow in the Earth's mantle with constraints from mineral physics and surface observations. Geophysical Journal International 167(3):1461-1481.

6. Forte AM, Dziewonski AM, Woodward RL (2013) A spherical Structure of the Mantle, Tectonic Plate Motions, Nonhydrostatic Geoid, and Topography of the Core-Mantle Boundary. (American Geophysical Union), pp. 135-166.

7. Hager BH, Richards MA (1989) Long-wavelength variations in earth's geoid: Physical models and dynamical implications. Philosophical Transactions of the Royal Society of London. Series A, Mathematical and Physical Sciences 328(1599):309-327.

8. Mitrovica JX, Peltier WR (1991) On postglacial geoid subsidence over the equatorial oceans. Journal of Geophysical Research: Solid Earth 96(B12):20053-20071.

9. Kiefer WS, Hager BH (1992) Geoid anomalies and dynamic topography from convection in cylindrical geometry: applications to mantle plumes on Earth and Venus. Geophysical Journal International 108(1):198-214

10. Osei Tutu A, Sobolev SV, Steinberger B, Popov AA, Rogozhina I (2018) Evaluating the influence of plate boundary friction and mantle viscosity on plate velocities. Geochemistry, Geophysics, Geosystems 19(3):642-666.

11. Richards, Hager, Richards M.A. HB (1988) The earth's geoid and the large-scale structure of mantle convection. pp. 247-272.

12. Peltier WR (1996) Mantle viscosity and ice-age ice sheet topography. Science 273(5280):1359-1364.

13. Simons M, Hager BH (1997) Localization of the gravity field and the signature of glacial rebound. Nature 390(6659):500-504.

14. Ghosh A, Becker TW, Zhong SJ (2010) Effects of lateral viscosity variations on the geoid. Geophysical Research Letters 37(1).

15. Zhong S, Davies GF (1999) Effects of plate and slab viscosities on the geoid. Earth and Planetary Science Letters 170(4):487 - 496.

16. Christensen U (1988) Is subducted lithosphere trapped at the $670-\mathrm{km}$ discontinuity? Nature 336(6198):462-463.

17. Fukao $\mathrm{Y}$, Widiyantoro S, Obayashi M (2001) Stagnant slabs in the upper and lower mantle transition region. Reviews of Geophysics 39(3):291-323.

18. Hayes GP, et al. (2018) Slab2, a comprehensive subduction zone geometry model. Science 362(6410):58-61.

19. French S, Romanowicz B (2015) Broad plumes rooted at the base of the earth's mantle beneath major hotspots. Nature 525:95.
20. P. Moulik GE (2014) An anisotropic shear velocity model of the earth's mantle using normal modes, body waves, surface waves and long-period waveforms. Geophysical Journal International 199(3):1713-1738.

21. Steinberger B (2000) Slabs in the lower mantle - results of dynamic modelling compared with tomographic images and the geoid. Physics of the Earth and Planetary Interiors 118(3):241-257.

22. Lithgow-Bertelloni C, Richards MA (1998) The dynamics of cenozoic and mesozoic plate motions. Reviews of Geophysics 36(1):27-78.

23. Rudolph ML, Lekić V, Lithgow-Bertelloni C (2015) Viscosity jump in earth's mid-mantle. Science 350(6266):1349-1352.

24. Reigber C, et al. (2005) An earth gravity field model complete to degree and order 150 from grace: Eigen-grace02s. Journal of Geodynamics 39(1):1 - 10.

25. Karato Si (2008) Deformation of Earth Materials: An Introduction to the Rheology of Solid Earth. (Cambridge University Press)

26. van der Meer DG, Spakman W, van Hinsbergen DJJ, Amaru ML, Torsvik TH (2010) Towards absolute plate motions constrained by lower-mantle slab remnants. Nature Geoscience 3(1):36-40.

27. King SD (1995) Radial models of mantle viscosity: results from a genetic algorithm. Geophysical Journal International 122(3):725-734.

28. Liu X, Zhong S (2016) Constraining mantle viscosity structure for a thermochemical mantle using the geoid observation. Geochemistry, Geophysics, Geosystems 17(3):895-913.

29. ichiro Karato S, Wang Z, Liu B, Fujino K (1995) Plastic deformation of garnets: systematics and implications for the rheology of the mantle transition zone. Earth and Planetary Science Letters 130(1):13 - 30

30. Ricard Y, Vigny C, Froidevaux C (1989) Mantle heterogeneities, geoid, and plate motion: A monte carlo inversion. Journal of Geophysical Research: Solid Earth 94(B10):13739-13754.

31. Ohtani E, Yuan L, Ohira I, Shatskiy A, Litasov K (2018) Fate of water transported into the deep mantle by slab subduction. Journal of Asian Earth Sciences 167:2-10.

32. Lenardic A, et al. (2011) Continents, supercontinents, mantle thermal mixing, and mantle thermal isolation: Theory, numerical simulations, and laboratory experiments. Geochemistry, Geophysics, Geosystems 12(10)

33. Le Pichon X, Şengör AMC, Imren C (2019) Pangea and the lower mantle. Tectonics 38(10):3479-3504.

34. Karlsen KS, Conrad CP, Domeier M, Trønnes RG (2021) Spatiotemporal variations in surface heat loss imply a heterogeneous mantle cooling history. Geophysical Research Letters 48(6):e2020GL092119.

35. Wieczorek MA, Simons FJ (2005) Localized spectral analysis on the sphere. Geophysical Journal International 162(3):655-675.

36. Schmidt M, et al. (2007) Regional gravity modeling in terms of spherical base functions. Journal of Geodesy 81(1):17-38.

37. Baur O, Sneeuw N (2011) Assessing greenland ice mass loss by means of point-mass modeling: a viable methodology. Journal of Geodesy 85(9):607-615

38. Harig C, Simons FJ (2012) Mapping greenland's mass loss in space and time. Proceedings of the National Academy of Sciences 109(49):19934-19937.

39. Bevis M, et al. (2019) Accelerating changes in ice mass within greenland, and the ice sheet's sensitivity to atmospheric forcing. Proceedings of the National Academy of Sciences 116(6):1934-1939.

40. Han SC, Simons FJ (2008) Spatiospectral localization of global geopotential fields from the gravity recovery and climate experiment (grace) reveals the coseismic gravity change owing to the 2004 sumatra-andaman earthquake. Journal of Geophysical Research: Solid Earth 113(B1).

41. Simons FJ, Dahlen FA, Wieczorek MA (2006) Spatiospectral concentration on a sphere. SIAM Review 48(3):504-536.

42. Simons FJ (2010) Slepian functions and their use in signal estimation and spectral analysis.

43. Osei Tutu A, Harig C (2022) Regional mantle viscosity constraints for north america revea upper mantle strength differences across the continent. Solid Earth Discussions 2022:1-37.

44. Dziewonski AM, Anderson DL (1981) Preliminary reference earth model. Physics of the Earth and Planetary Interiors 25(4):297-356.

45. Simmons NA, Forte AM, Boschi L, Grand SP (2010) Gypsum: A joint tomographic mode of mantle density and seismic wave speeds. Journal of Geophysical Research: Solid Earth 115(B12).

46. Forte AM, Peltier R (1991) Viscous flow models of global geophysical observables: 1. forward problems. Journal of Geophysical Research: Solid Earth 96(B12):20131-20159.

47. Sambridge M, Bodin T, Gallagher K, Tkalčić H (2013) Transdimensional inference in the geosciences. Philosophical Transactions of the Royal Society A: Mathematical, Physical and Engineering Sciences 371(1984):20110547.

48. Green PJ (1995) Reversible jump Markov chain Monte Carlo computation and Bayesian model determination. Biometrika 82(4):711-732.

49. Malinverno A (2002) Parsimonious Bayesian Markov chain Monte Carlo inversion in a nonlinear geophysical problem. Geophysical Journal International 151(3):675-688. 


\title{
1 \\ Supplementary Information for \\ 2 Variations in Earth's 1D viscosity structure in different tectonic

\author{
Osei Tutu, Anthony*1 and Harig, Christopher ${ }^{\dagger 1}$ \\ ${ }^{1}$ Department of Geosciences, University of Arizona, Tucson
}

\section{Additional Figures}
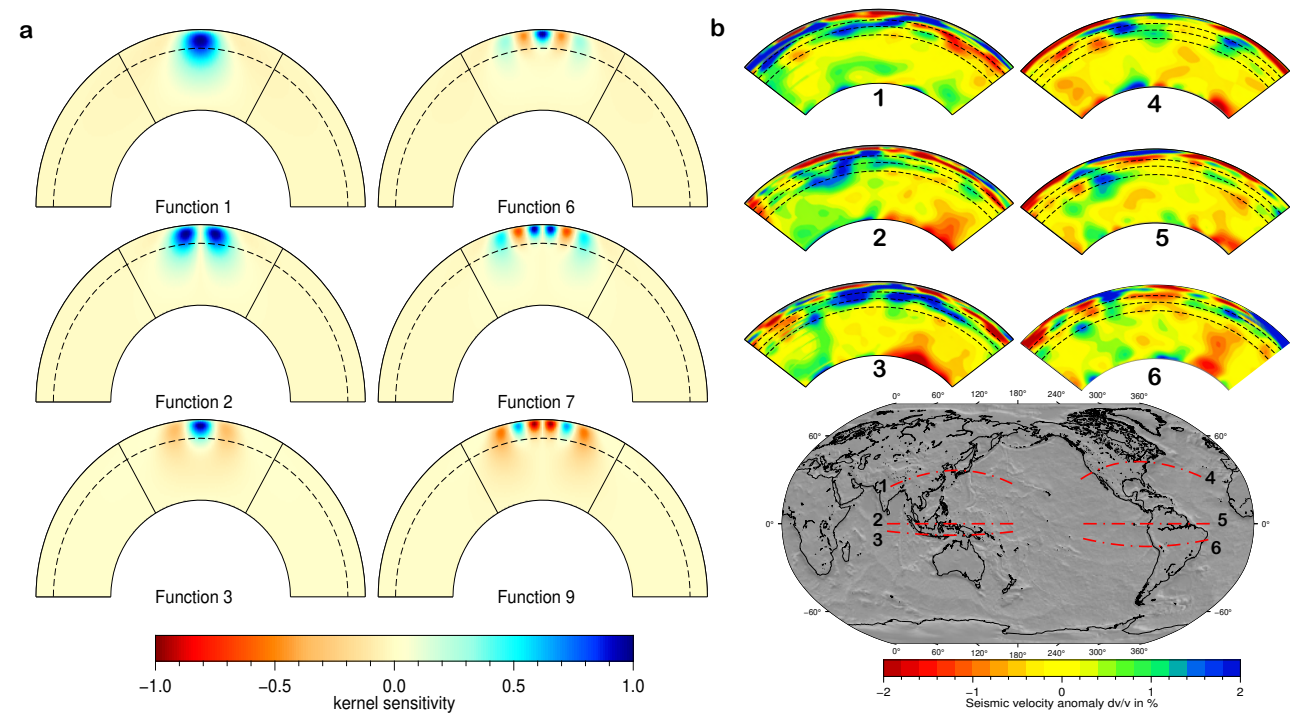

Figure 1: a) Local sensitivity dynamic geoid kernels with an iso-viscous mantle. Shown is a cross-section along $0^{\circ}$ and $180^{\circ}$ in the northern hemisphere from the surface to the core mantle boundary. The kernels have azimuthal dependence and as such will have different manifestations at different azimuths. The kernels are localized to a $50^{\circ}$ spherical cap, denoted by black lines connecting the surface to the core mantle boundary. Here, the bandwidth of the basis is $l=30$ showing short wavelength effects compare to the Fig. $1 \mathrm{~b}$ in the main text $l=9$. Functions are ranked by concentration within the region, and shown are functions $1,2,3,4,7$, and 9 . The kernels are normalized by their maximum absolute value. b) Vertical cross sections of seismic tomography model ${ }^{2}$ showing slabs distribution within our Pacific local mantle.

*oseitutu@arizona.edu

${ }^{\dagger}$ charig@arizona.edu 

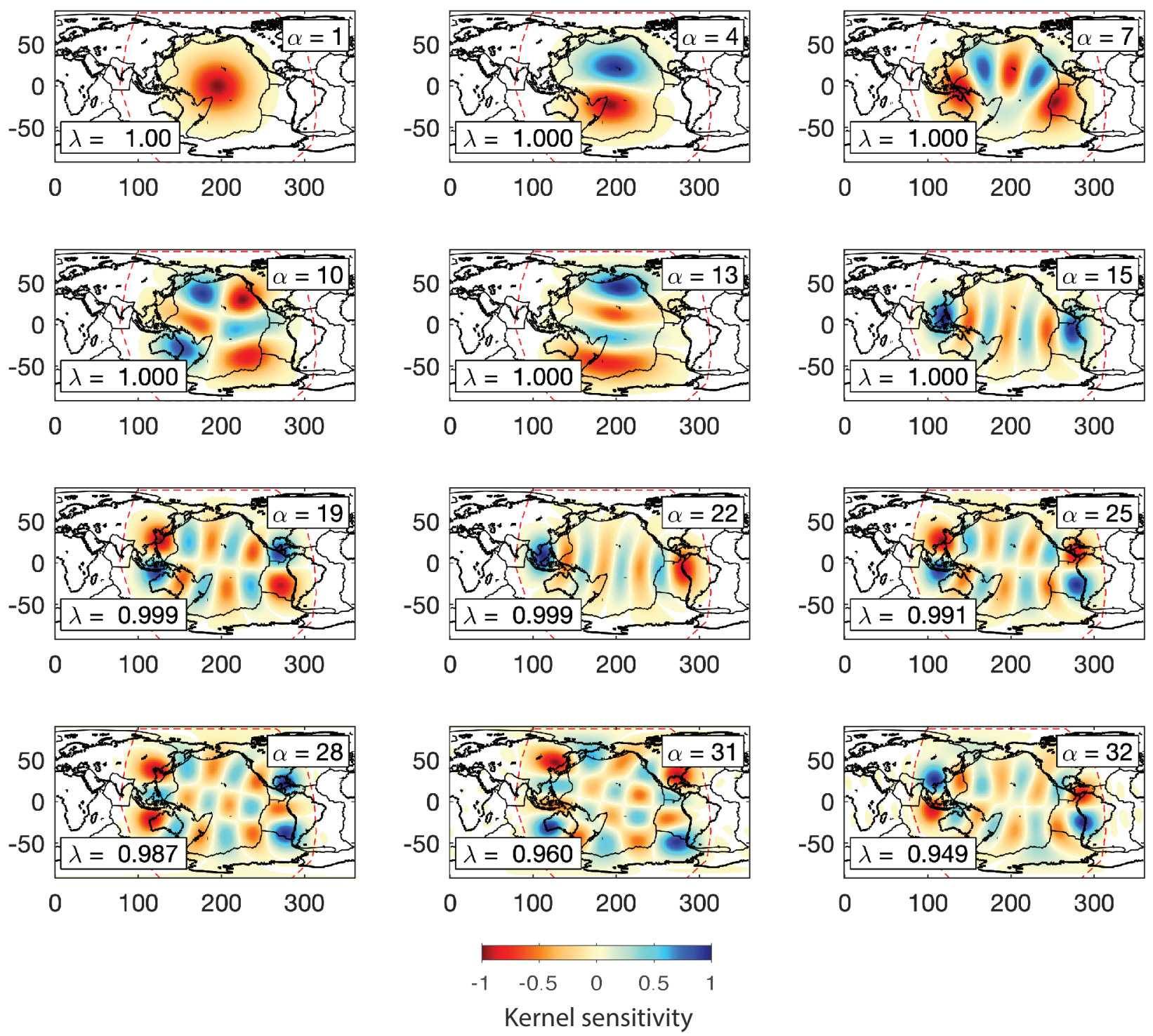

Figure 2: Our Pacific hemisphere showing maps of synthesized concentrated Slepian eigenfunctions. $\alpha$ indicates the eigenfunction number and rank while the eigenvalue concentration factors are labeled as $\lambda$. 

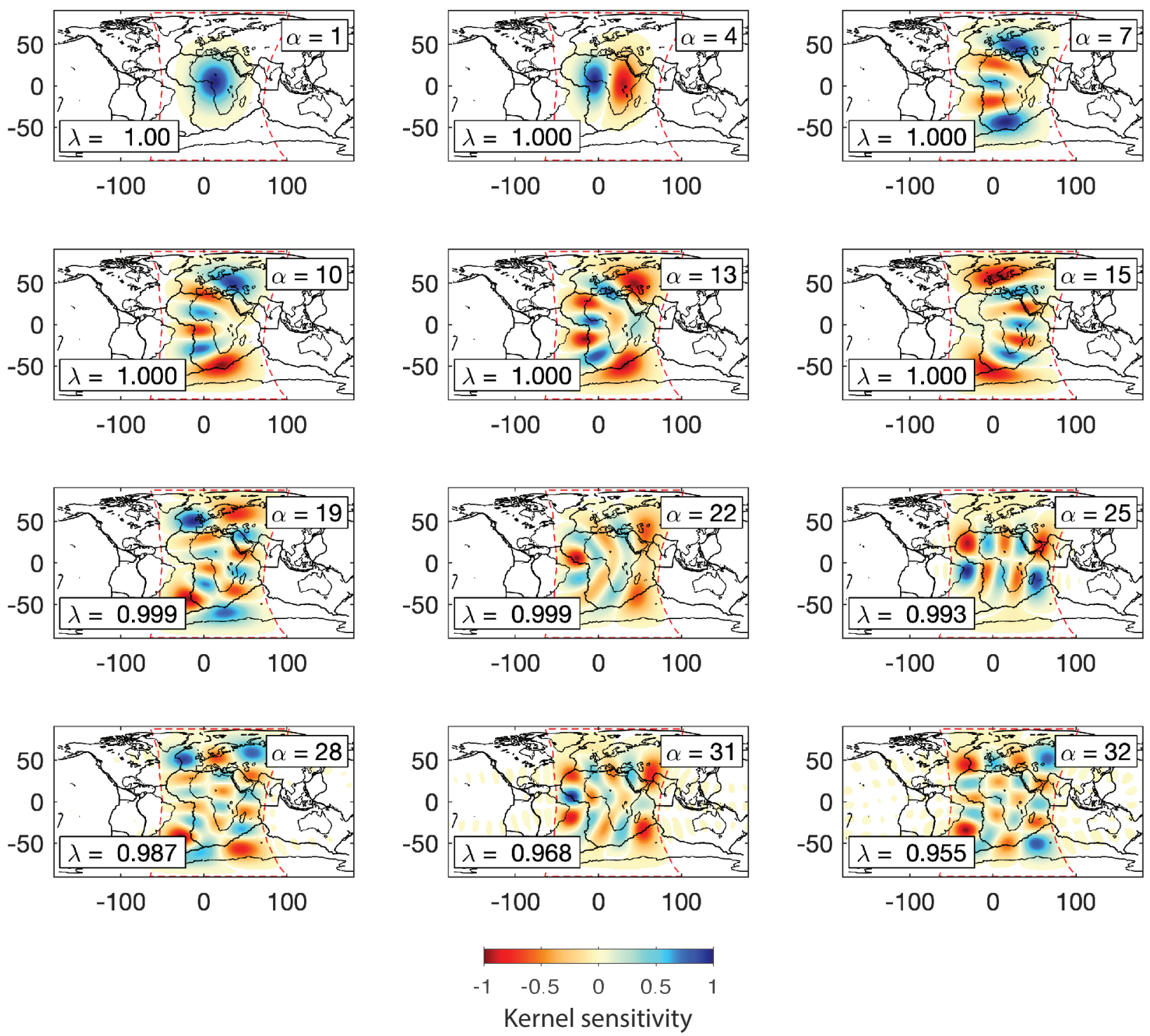

Figure 3: Maps of synthesized concentrated Slepian eigenfunctions for our Atlantic region. $\alpha$ indicates the eigenfunction number and rank while the eigenvalue concentration factors are labeled as $\lambda$. The maps are centered in the African hemisphere. 


\section{Global viscosity solutions}

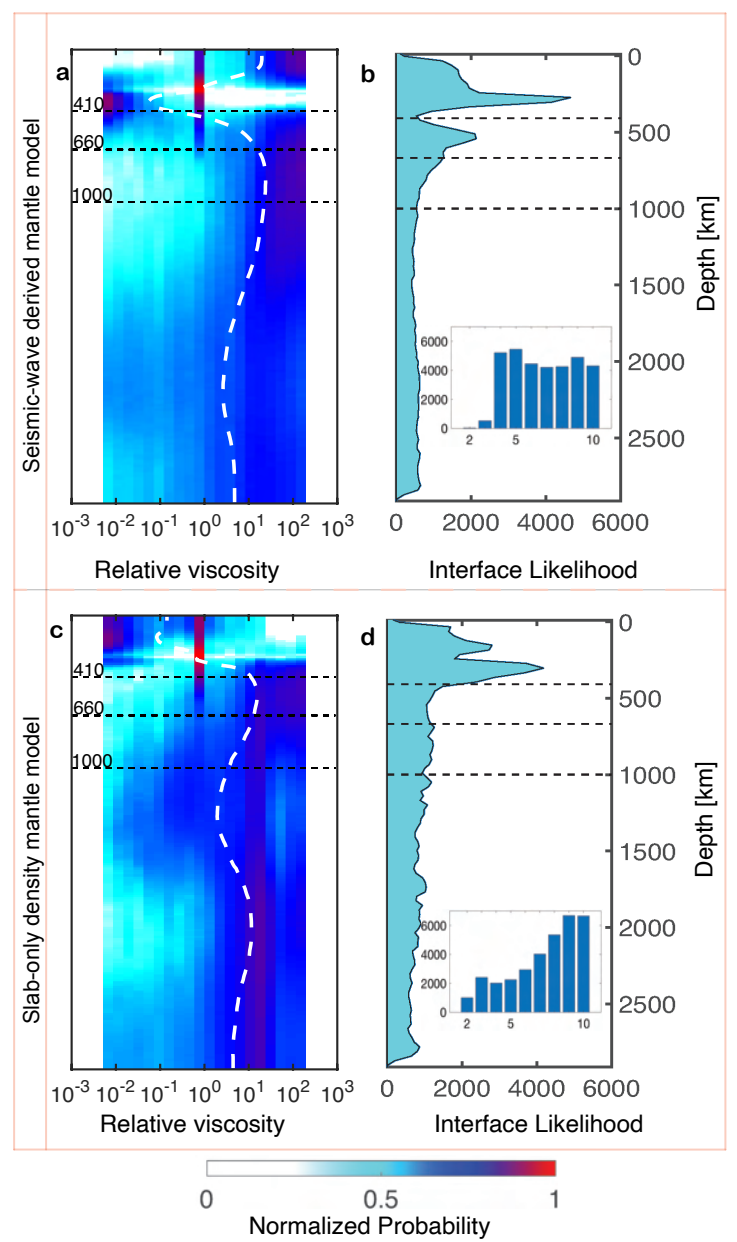

Figure 4: a) Layered mantle viscosity solutions from large-scale global mantle flow for spherical harmonics degrees $l=4$ to 9 using a seismically-derived mantle model ${ }^{2}$ considering $\frac{d \ln \rho}{d \ln V_{s}}=$ 0.35 (a-b) and plate reconstruction slab-only mantle model ${ }^{5}$ (c-d). Panels a and $\mathbf{c}$ show 2D histograms of the posterior probability distributions of viscosity with depths expressed as normalized probability and the white dash lines giving the mean relative viscosity profiles. Panels $\mathbf{b}$ and $\mathbf{d}$ show resulting mantle-viscosity interface distributions with the corresponding inset histograms giving the number of layers for each solution. 

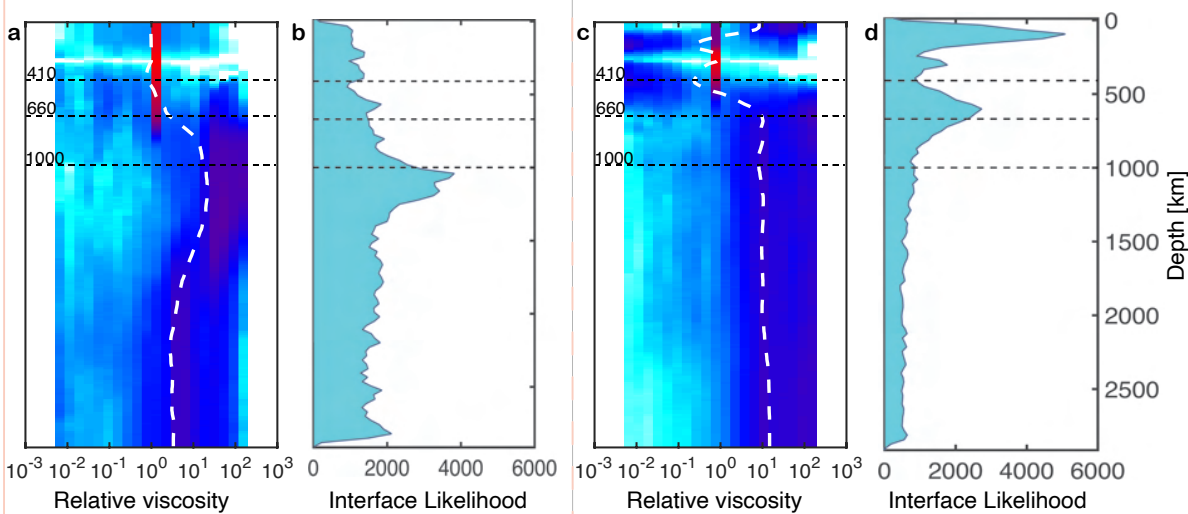

Relative viscosity Interface Likelihood Relative viscosity Interface Likelihood
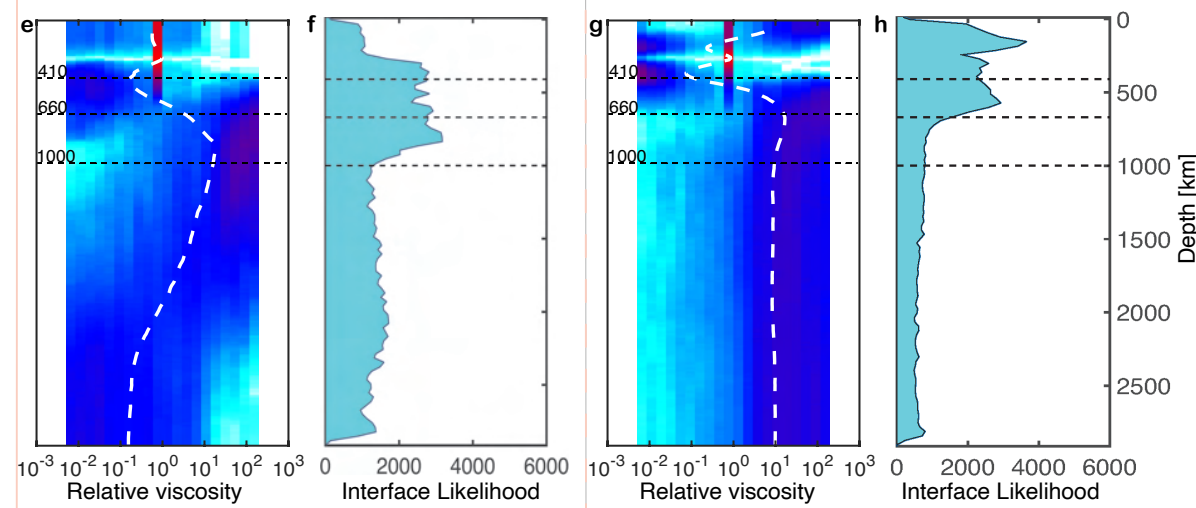

0

Normalized Probability

Figure 5: Global layered mantle viscosity solutions from large-scale mantle flow using seismicallyderived mantle model $\mathrm{S} 362 \mathrm{ANI}+\mathrm{M}^{3}$ considering $\frac{d \ln \rho}{d \ln V_{s}}=0.35$ (a-d) and depth dependent $\delta \rho=\frac{d \ln \rho}{d \ln V_{s}}$ from $^{4}$ (e-h) seismic velocity-to-density scaling. Plots showing (a, c, e and g) 2D histogram of the posterior probability distributions of viscosity with depths expressed as normalized probability and the white dash lines giving the mean relative viscosity profiles. Panels $\mathrm{b}, \mathrm{d}, \mathrm{f}$, and $\mathrm{h}$ show resulting mantle-viscosity interfaces. The left and right halves of the figure represent the inversion solutions for spherical harmonics degrees $l=2$ to 3 and $l=4$ to 9 respectively. 


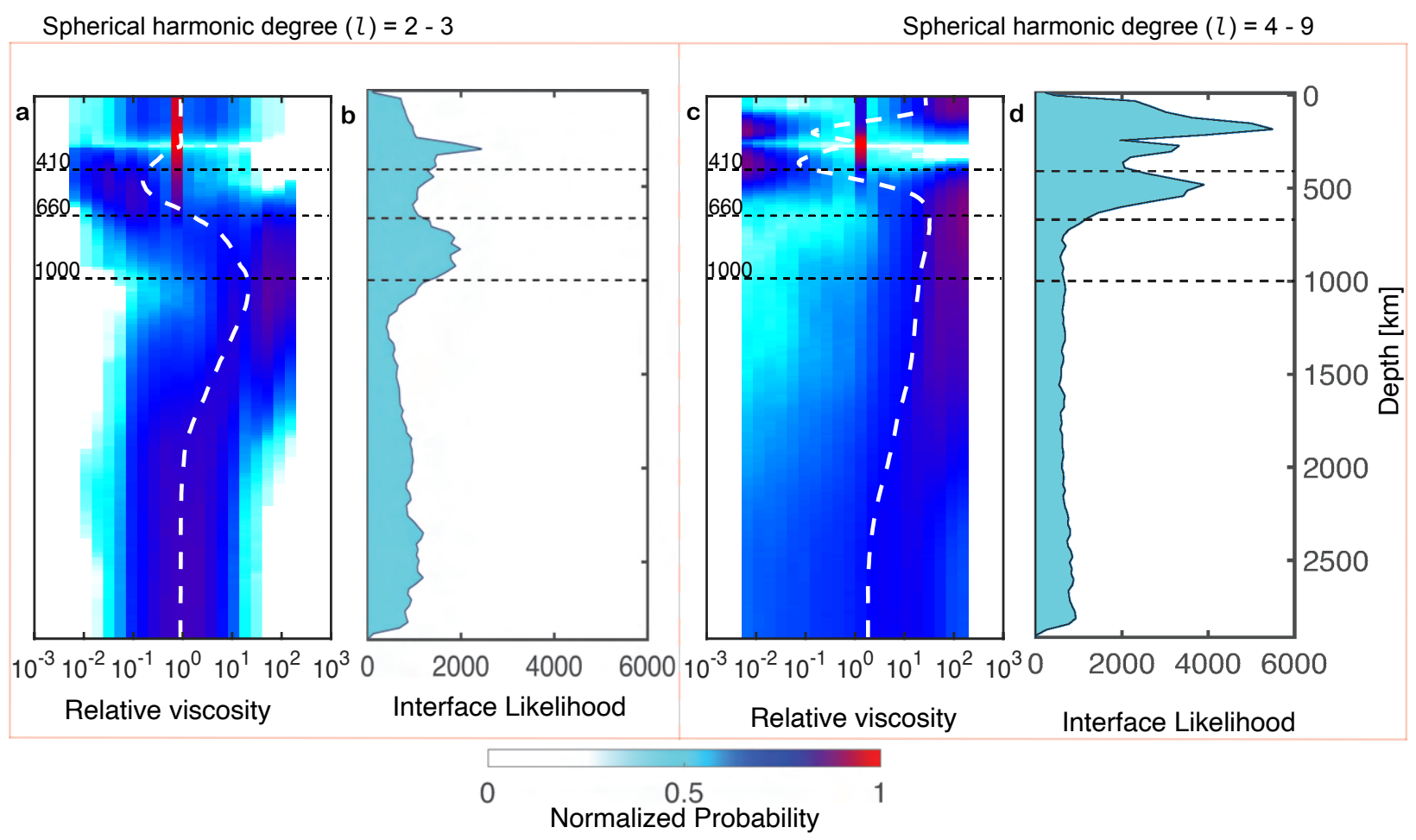

Figure 6: Global layered mantle viscosity solutions from large-scale mantle flow using seismicallyderived mantle model SEMUCB-WM1 ${ }^{2}$ with depth-dependent $\delta \rho=\frac{d \ln \rho}{d \ln V_{s}}$ from Simmons et al., ${ }^{4}$ velocity-to-density scaling. Panels a and c show 2D histogram of the posterior probability distributions of viscosity with depths expressed as normalized probability and the white dash lines giving the mean relative viscosity profiles. Panels $\mathrm{b}$ and $\mathrm{d}$ show resulting mantle-viscosity interfaces distributions. The left and right halves of the figure represent the inversion solutions for spherical harmonics degrees $l=2$ to 3 and $l=4$ to 9 respectively. 
8 Local viscosity solutions 


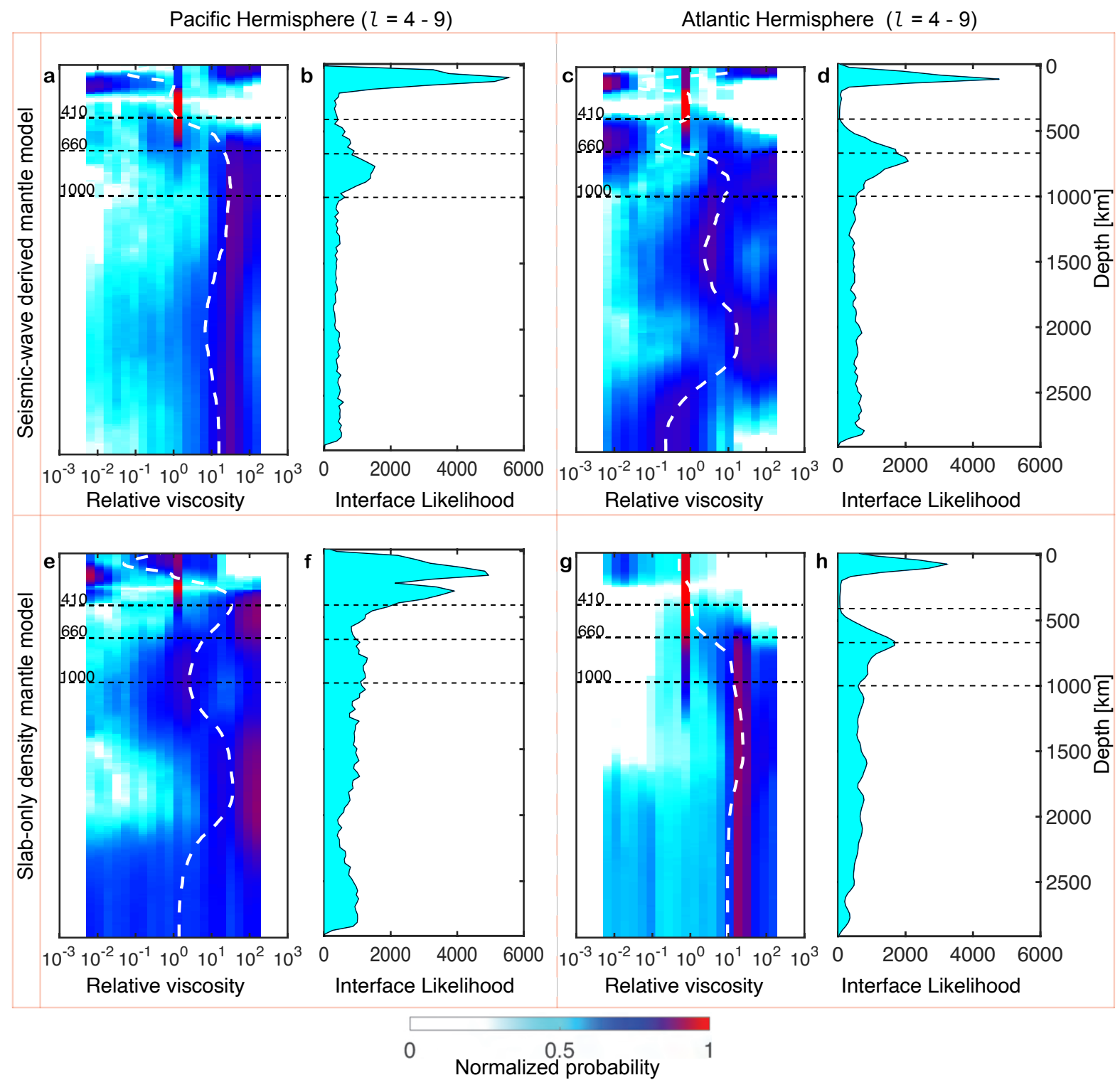

Figure 7: Intermediate-wavelength $(l=4-9)$ local viscosity solutions based on regional mantle models from $(\mathrm{a}-\mathrm{d})$ seismically-derived mantle model ${ }^{2}$ considering $\frac{d \ln \rho}{d \ln V_{s}}=0.35$ and $(\mathrm{e}-\mathrm{h})$ plate reconstruction slab-only mantle model Steinberger et al., ${ }^{5}$ for the Pacific and Atlantic regions. Plots a, c, e, and g show 2D histograms of the posterior probability distributions of viscosity with depth, expressed as normalized probability. White dash lines give the mean relative viscosity profiles. Panels b, d, f, and h show resulting mantle-viscosity interfaces distributions. The left and right halves of the figure represent the inversion solutions for spherical harmonics degrees $l=4-9$ for the Pacific and Atlantic regions respectively. 


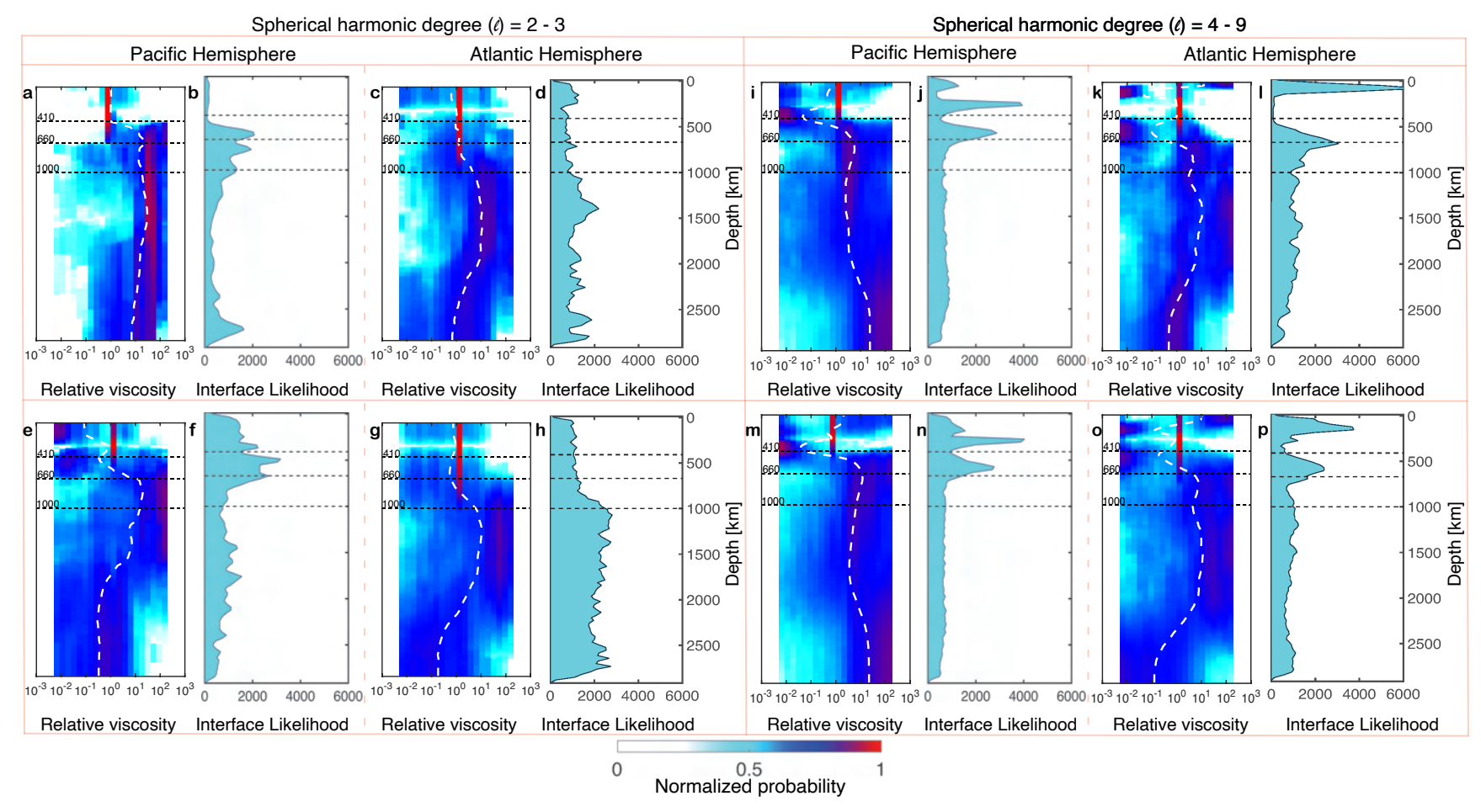

Figure 8: Local viscosity solutions based on regional mantle models from seismically-derived mantle models from $S 362 A N I+M^{3}$ for the Pacific $(\mathrm{a}-\mathrm{h})$ and Atlantic (i-p) regions with constant $\frac{d \ln \rho}{d \ln V_{s}}=0.35$ (top row) and depth-dependent ${ }^{4}$ (bottom row) seismic velocity-to-density scalings. Plots showing (a, c, i, k, e, g, m, and o) 2D histogram of the posterior probability distributions of viscosity with depths expressed as normalized probability and the white dashed lines giving the mean relative viscosity profiles. Panels $\mathrm{b}, \mathrm{d}, \mathrm{j}, \mathrm{l}, \mathrm{f}, \mathrm{h}, \mathrm{n}$, and $\mathrm{p}$ show resulting mantle-viscosity interfaces probabilities. The left and right halves of the figure represent the inversion solutions for spherical harmonics degrees $l=2$ to 3 and $l=4$ to 9 respectively. 
a

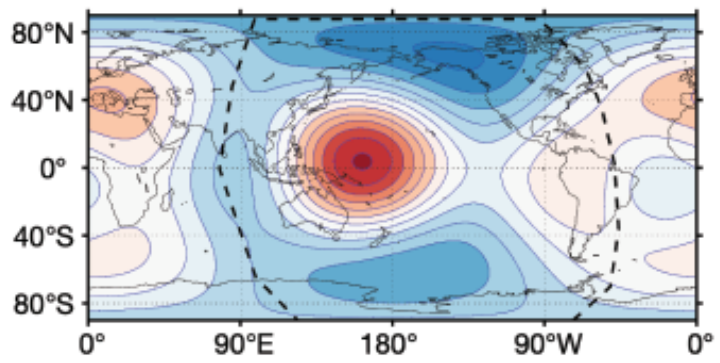

C

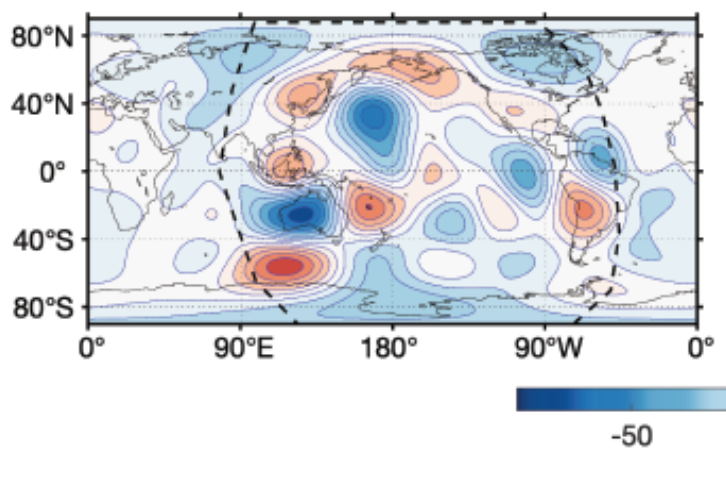

b

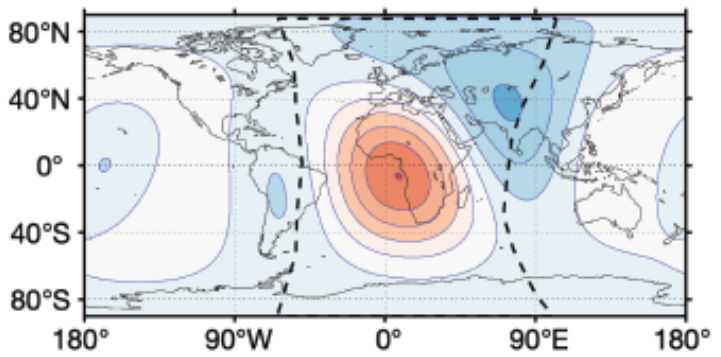

d

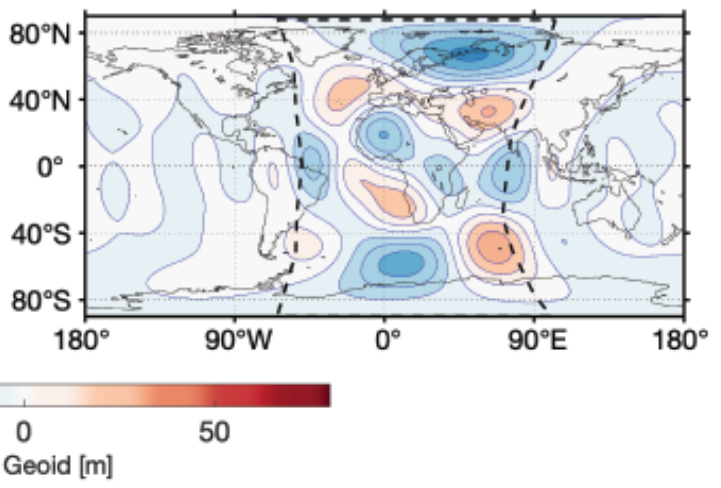

Figure 9: Maps showing ensemble average of local geoid signals ( $l=2$ to 3 ) for (a) Pacific region and (b) Atlantic region resulting from the local radial viscosity inversions, based on Seismic model SEMUCB-WM12 ${ }^{2}$ with constant seismic velocity-to-density scaling factor 0.35 . Similar ensemble average local geoid maps of $l=4$ to 9 for the (c) Pacific (d) Atlantic regions. The black dash outlines are the Pacific ( $a$ and $c$ ) and Atlantic (b and d) regional boundaries for our Slepian localization techniques. 


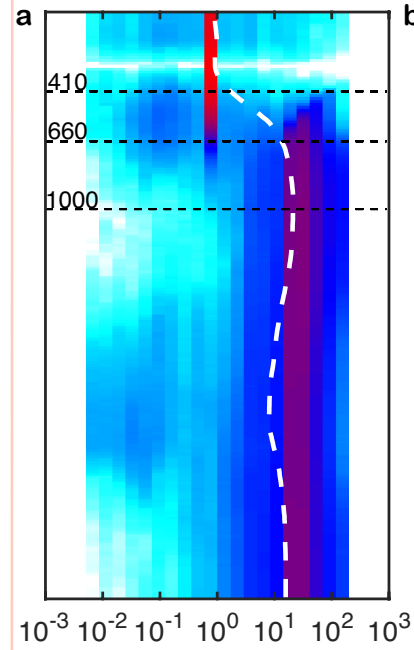

Relative viscosity

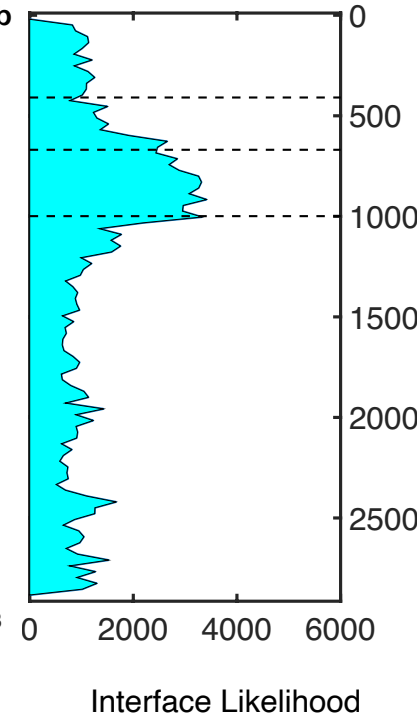

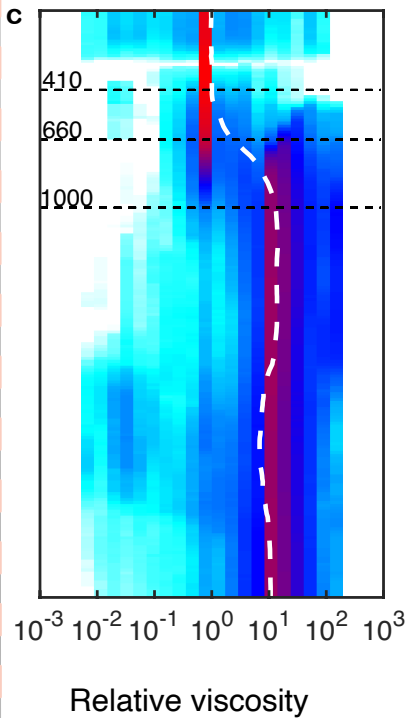

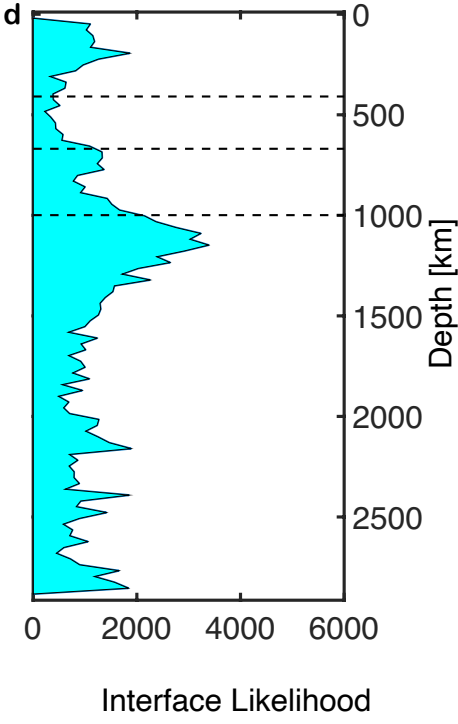

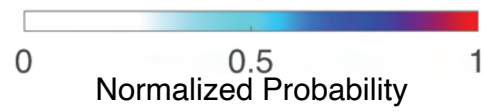

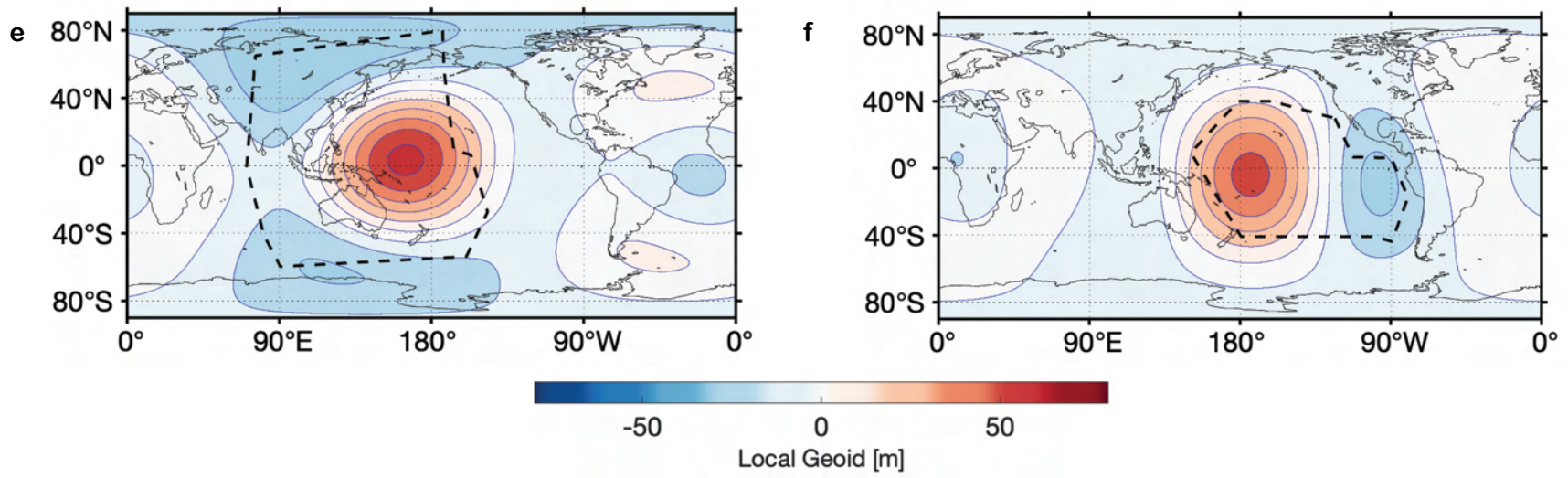

Figure 10: Long-wavelength $(l=2-3)$ local viscosity solutions based on regional seismicallyderived mantle model from SEMUCB-WM1 ${ }^{2}$ for (a-b) Western and (c-d) Central Pacific. Plots a, and c show 2D histograms of the posterior probability distributions of viscosity with depth, expressed as normalized probability. The white dash lines give the mean relative viscosity profiles. Panels $\mathrm{b}$, and $\mathrm{d}$ show the resulting mantle-viscosity interfaces distributions. Maps showing ensemble average of local geoid signals ( $l=2$ to 3$)$ for (e) Western and (f) Central Pacific resulting from the local radial viscosity inversions, based on Seismic model SEMUCB$\mathrm{WM}^{2}$ with constant seismic velocity-to-density scaling factor 0.35 . The black dash outlines are the regional boundaries for our Slepian localization techniques. 
a

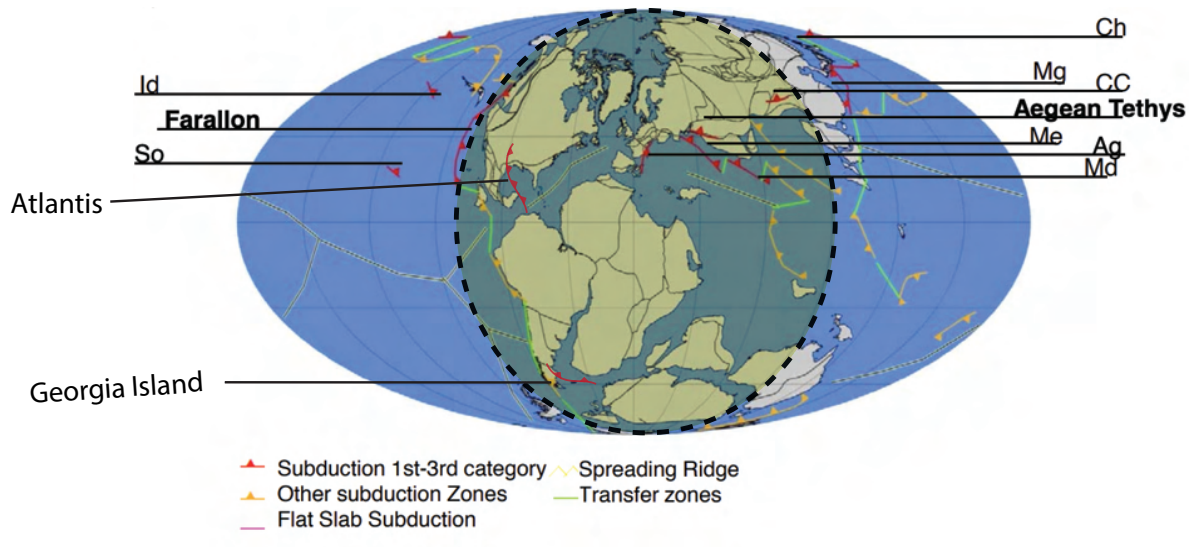

b

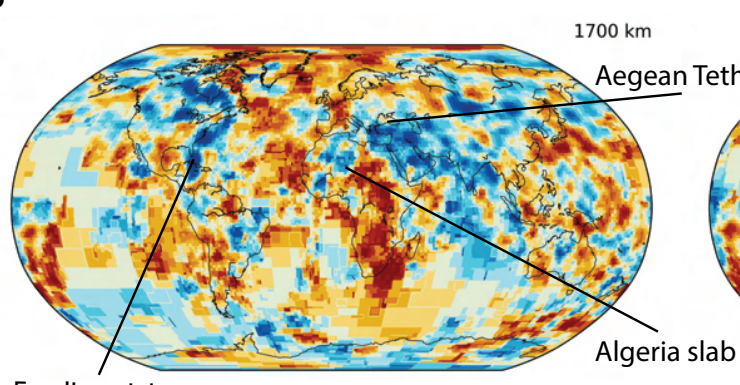

C

Farallon slab

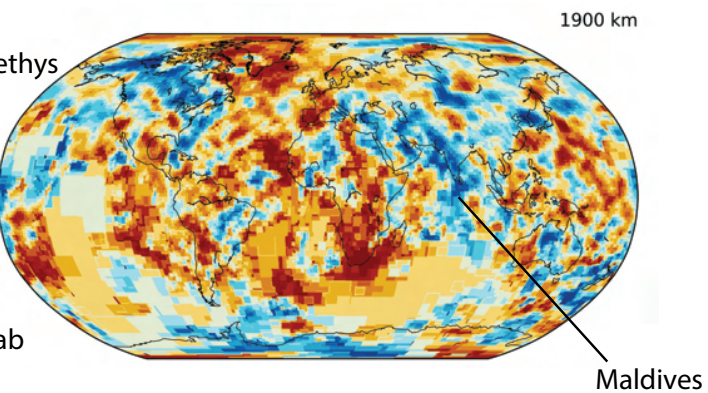

d

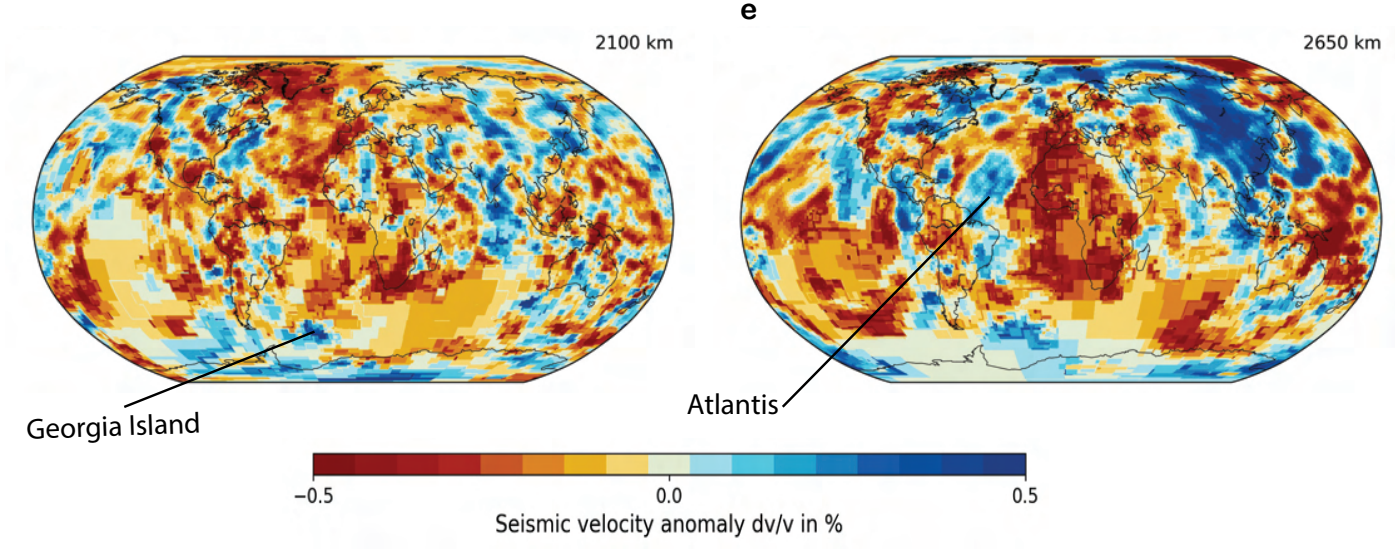

Figure 11: a) Paleo-Geographic map with the longitudinal position of past oceanic subduction zones modifies after van der Meer et al., ${ }^{6}$ depicting the likely position of the Ag - Algeria, CC - Central China, Ch - Chukchi, Id - Idaho, Me - Mesopotamia, At - Atlantis, Mg - Mongolia, GI Georgia Islands, So - Socorro, Md - Maldives slabs. The overlying yellow shade with dash black outline shows the approximate Atlantic region for the local viscosity inversion with our spatiospectral localization technique. b-e) Seismic tomographic depth slices ${ }^{1}$ showing mid-tolower mantle slabs remnants in the Atlantic/African hemisphere based on the analysis of van der Meer et al., ${ }^{6 ; 7}$ 


\section{References}

[1] M.L. Amaru. Global travel time tomography with 3-d reference models. Geol. Ultraiect, 274: 174, 2007. URL hhttps://dspace.library.uu.nl/handle/1874/19338.

[2] Scott French and Barbara Romanowicz. Broad plumes rooted at the base of the earth's mantle beneath major hotspots. Nature, 525:95, 2015. doi: https://doi.org/10.1038/nature14876. URL https://www.nature.com/articles/nature14876supplementary-information.

[3] G. Ekström P. Moulik. An anisotropic shear velocity model of the earth's mantle using normal modes, body waves, surface waves and long-period waveforms. Geophysical Journal International, 199:1713-1738, 2014.

[4] Nathan A. Simmons, Alessandro M. Forte, Lapo Boschi, and Stephen P. Grand. Gypsum: A joint tomographic model of mantle density and seismic wave speeds. Journal of Geophysical Research: Solid Earth, 115(B12), 2010. doi: 10.1029/2010JB007631. URL https://agupubs. onlinelibrary.wiley.com/doi/abs/10.1029/2010JB007631.

[5] Bernhard Steinberger. Slabs in the lower mantle - results of dynamic modelling compared with tomographic images and the geoid. Physics of the Earth and Planetary Interiors, 118 (3):241 - 257, 2000. ISSN 0031-9201. doi: https://doi.org/10.1016/S0031-9201(99)00172-7. URL http://www.sciencedirect.com/science/article/pii/S0031920199001727.

[6] Douwe G. van der Meer, Wim Spakman, Douwe J. J. van Hinsbergen, Maisha L. Amaru, and Trond H. Torsvik. Towards absolute plate motions constrained by lower-mantle slab remnants. Nature Geoscience, 3(1):36-40, 2010. ISSN 1752-0908. doi: https://doi.org/10. 1038/ngeo708.

[7] Douwe G. van der Meer, Douwe J.J. van Hinsbergen, and Wim Spakman. Atlas of the underworld: Slab remnants in the mantle, their sinking history, and a new outlook on lower mantle viscosity. Tectonophysics, 723:309-448, 2018. ISSN 0040-1951. doi: https://doi.org/10.1016/j.tecto.2017.10.004. URL https://www.sciencedirect.com/science/ article/pii/S0040195117304055. 